\title{
Inégalites socio-spatiales de risque d'accident en tant que piéton : un cumul de facteurs individuels et contextuels?
}

\section{Sylvanie Godillon et Julie Vallée}

\section{(2) OpenEdition}

\section{Journals}

Édition électronique

URL : http://journals.openedition.org/rfst/447

DOI : $10.4000 /$ rfst. 447

ISSN : 2492-3672

Éditeur

Espaces et SOciétés (UMR 6590)

Référence électronique

Sylvanie Godillon et Julie Vallée, «Inégalites socio-spatiales de risque d'accident en tant que piéton: un cumul de facteurs individuels et contextuels? ", Revue francophone sur la santé et les territoires [En ligne], Miscellanées, mis en ligne le 13 mai 2015, consulté le 06 avril 2021. URL : http:// journals.openedition.org/rfst/447 ; DOI : https://doi.org/10.4000/rfst.447

Ce document a été généré automatiquement le 6 avril 2021.

La Revue francophone sur la santé et les territoires est mise à disposition selon les termes de la Licence Creative Commons Attribution - Pas d'Utilisation Commerciale - Partage dans les Mêmes Conditions 4.0 International. 


\title{
Inégalites socio-spatiales de risque d'accident en tant que piéton : un cumul de facteurs individuels et contextuels?
}

\author{
Sylvanie Godillon et Julie Vallée
}

\section{Introduction}

\section{Des inégalités socio-spatiales devant le risque routier}

1 En 2004, la Journée mondiale de la Santé était consacrée à la sécurité routière. Les accidents de la circulation ont en effet des répercussions importantes sur la santé des populations, notamment des jeunes adultes pour qui ils constituent la première cause de décès selon l'Organisation Mondiale de la Santé.

2 Comme pour d'autres indicateurs de santé, le risque routier se décline différemment selon les groupes sociaux. Des travaux de recherche ont ainsi souligné des taux d'accidents particulièrement élevés pour les classes populaires (Van Beeck et al., 1991) et des risques d'accident de 20 à $30 \%$ supérieurs pour les enfants de familles d'ouvriers que pour les enfants de classes intermédiaires (Hasselberg, et al., 2001). Les inégalités sociales devant le risque d'accident se sont accentuées entre 1981 et 1991 avec une diminution plus forte des taux d'accidents pour les enfants issus des «classes non manuelles » que pour les enfants issus des «classes manuelles » (Roberts \& Power, 1996).

3 Au-delà des caractéristiques sociales des individus, d'autres études se sont concentrées sur les caractéristiques des quartiers de résidence des personnes impliquées dans un accident de la route. A Montréal (Canada), le risque d'accident de la route des enfants de moins de 15 ans est quatre fois plus élevé pour les enfants des quartiers pauvres que pour ceux des quartiers riches (Dougherty et al., 1990). Ces résultats se vérifient 
également pour la population adulte en Ecosse avec des risques d'accidents plus élevés pour les habitants des zones défavorisées que pour ceux des zones plus aisées (Abdalla et al., 1997). De même, les habitants de Zones Urbaines Sensibles dans l'agglomération lilloise ou dans le département du Rhône en France enregistrent un plus fort risque d'accident que les habitants de quartiers plus favorisés (Fleury et al., 2010 ; Haddak et al., 2012).

\section{Les risques d'accident piéton}

4 Certains usagers de la route comme les piétons mais aussi les cyclistes et les motocyclistes sont particulièrement vulnérables. L'Observatoire National de la Sécurité Routière montre que si le risque d'être tué (par heure passée de trajet) est 1,2 fois moins élevé pour un piéton que pour un automobiliste, le risque d'être gravement blessé est 1,6 fois plus élevé que pour un automobiliste (ONISR, 2014). Dans un contexte de hausse de la part modale de la marche en ville, une meilleure compréhension des facteurs de risque d'accident piéton constitue donc un enjeu important pour l'amélioration de la sécurité routière.

5 A la différence de recherches portant sur les caractéristiques environnementales des lieux des accidents piétons (Morency et al., 2011; Cloutier, 2014), des recherches ciblent plus spécifiquement les caractéristiques des quartiers de résidence des impliqués. Au Canada, les différences de risques d'accidents entre quartiers sont plus fortes pour les piétons que pour les cyclistes (Dougherty et al., 1990). Un lien a été établi entre les caractéristiques sociales de la population du quartier de résidence (définies à partir du taux de chômage, de la part des familles monoparentales, de la part des étrangers, ou encore du nombre moyen d'enfants dans les famille) et le risque d'accident en tant que piéton (Edwards et al., 2006 ; Reimers et al., 2008 ; Hasselberg, et al., 2001).

6 Parallèlement, des recherches ont mis en évidence l'importance des caractéristiques de l'habitat résidentiel. Il a été observé que les enfants vivant dans des immeubles ont 5,5 fois plus de risque d'accident en tant que piéton que les enfants vivant dans des maisons individuelles (Mueller, et al., 1990). Le rôle de la sur-occupation des logements dans le risque d'accident piéton des enfants a aussi été mis en évidence avec un risque accru d'accident piéton pour les enfants vivant dans des logements sur-occupés (Christoffel, et al., 1996). Une des hypothèses avancée est que cette sur-occupation est associée à une présence de familles nombreuses et que les enfants des familles nombreuses enregistraient un risque d'accident piéton élevé (Anderson, et al., 1998). Ces résultats sont anciens et ne concernent que les risques des enfants. Ils permettent néanmoins de pointer l'importance de la forme urbaine de l'espace résidentiel dans l'explication du risque d'accident piéton. Une autre dimension du contexte résidentiel à prendre en compte est l'intensité du trafic routier. On peut en effet se demander si de forts niveaux de trafics routiers dans l'environnement résidentiel sont liés à un risque plus élevé d'accident piéton pour les populations résidentes, quelque soit le lieu où se produit l'accident. A Montréal, le nombre de rues traversées par les enfants lors d'un déplacement à pied est inversement proportionnel au statut socio-économique (Macphersen et al., 1998). Ces résultats révèlent des inégalités sociales d'exposition au trafic, mais ne renseignent pas les niveaux de trafic dans le quartier de résidence. D'autres études qualitatives indiquent que les habitants de quartiers défavorisés sont 
plus exposés aux risques liés au trafic. Par exemple, les discours de parents d'enfants âgés de 9 à 14 ans vivant dans dix quartiers défavorisés en Angleterre soulignent le danger que représente un important trafic routier dans le quartier de résidence pour les enfants qui jouent dans la rue (Christie et al., 2007).

7 Les inégalités sociales quant au risque d'être impliqué dans un accident en tant que piéton sont aussi à mettre en regard avec les différenciations sociales dans l'utilisation des modes de transport. A caractéristiques identiques (niveau d'étude, âge, motorisation), les habitants des quartiers défavorisés sont en effet généralement moins mobiles. Leur nombre de déplacements quotidiens est plus faible et leur mobilité se réalise davantage à pied et en transport en commun, et nettement moins en voiture (Quetelard, 1998; Fol, 2009). En Grande-Bretagne, les enfants issus d'un ménage ne possédant pas de voiture marchent davantage que les autres, ce qui explique des différences de risque par mode de transport et peut contribuer aux différences sociales observés dans les taux d'accidents piétons (Sonkin et al., 2006).

\section{Objectif de l'étude}

8 A partir de données portant sur les accidents de la route dans l'agglomération lilloise (France), cet article s'intéresse au profil des individus impliqués dans un accident en tant que piéton en le comparant à celui des individus impliqués dans un accident avec un autre mode de transport (conducteur ou passager de voiture particulière, cycliste, usager de transport en commun, etc.). L'idée est d'analyser les inégalités socio-spatiales de risque d'accident piéton: les habitants de quartiers défavorisés sont-ils plus impliqués dans un accident de la route en tant que piéton que les habitants de quartiers aisés? A notre connaissance, aucune recherche portant sur les risques d'accident piéton n'a cherché à considérer de façon conjointe les facteurs individuels et les facteurs contextuels des quartiers de résidence. Une fois les caractéristiques individuelles prises en compte, quelles sont les variables de l'environnement résidentiel qui sont associées à un risque accru d'accident piéton? Dans quelle mesure les caractéristiques du quartier de résidence des individus (en termes d'environnement social, de logement ou de trafic) sont-elles associées au risque d'être impliqué dans un accident de la route en tant que piéton?

\section{Méthodologie}

\section{Terrains d'étude : 18 quartiers de l'agglomération lilloise}

9 Cette recherche se concentre sur les accidents de la route qui ont impliqué les habitants de neuf quartiers défavorisés et de neuf quartiers favorisés de l'agglomération lilloise. Les « quartiers » sont ici définis comme des assemblages d'IRIS 1 .

Un IRIS est considéré comme défavorisé s'il appartient à une Zone Urbaine Sensible (ZUS). Ce découpage institutionnel identifie des territoires prioritaires dans le cadre de la politique de la ville du fait des difficultés qu'ils rencontrent, principalement en matière d'emploi et de chômage. Ce découpage s'appuie sur l'analyse de critères comme le taux de chômage, la proportion d'habitants de moins de 25 ans, la proportion d'habitants sans diplôme, et le potentiel fiscal de la commune. Dans l'agglomération 
lilloise, seize ZUS ont été identifiées par les pouvoirs publics. Elles regroupaient en 2006 plus de 190000 personnes, soit $17 \%$ de la population de l'agglomération lilloise. Pour notre étude, neuf de ces ZUS (soit $60 \%$ de l'ensemble des habitants des ZUS de l'agglomération lilloise) ont été choisies selon plusieurs critères. D'une part, l'échantillon compte des quartiers défavorisés plus ou moins éloignés des centres de l'agglomération lilloise que sont Lille, Roubaix et Tourcoing. D'autre part, nous avons sélectionné des quartiers avec des morphologies urbaines contrastées (quartiers d'habitat ancien et de grands ensembles) afin d'appréhender l'influence des caractéristiques urbaines sur la mobilité et l'insécurité routière.

11 Les accidents de la route qui ont impliqué les habitants de ces quartiers défavorisés sont comparés à ceux qui ont impliqué les habitants de neuf «quartiers de comparaison ». Ces quartiers sont localisés à proximité des quartiers défavorisés (Figure 1). Les critères de choix ont été la continuité spatiale avec les quartiers défavorisés sélectionnés et une composition sociodémographique différente.

12 Le risque d'accident en tant que piéton des habitants de ces 47 IRIS (103 431 habitants) inclus dans les neuf ZUS et les 32 IRIS différents des neuf quartiers de comparaison choisis (78 857 habitants) est étudié dans cet article.

13 La structure démographique et sociale de la population est différente entre les neuf quartiers défavorisés étudiés et les neuf quartiers de comparaison. Si la répartition entre les hommes et les femmes est identique (respectivement $48 \%$ et $52 \%$ de la population), la structure par âge diffère avec une plus forte proportion de moins de 18 ans dans les quartiers défavorisés ( $28 \%$ contre $22 \%$ dans les quartiers de comparaison). Les actifs ne représentent que $20 \%$ de la population dans les quartiers défavorisés contre 30 \% dans les quartiers de comparaison. Le taux de chômage en 2006 est de 28 \% dans les quartiers défavorisés contre $13 \%$ dans les quartiers de comparaison et la part d'ouvriers de $36 \%$ contre $21 \%$ (d'après le Recensement Général de la Population, INSEE, 2007). 
Figure 1 : Localisation des 18 quartiers étudiés dans Lille Métropole Communauté Urbaine

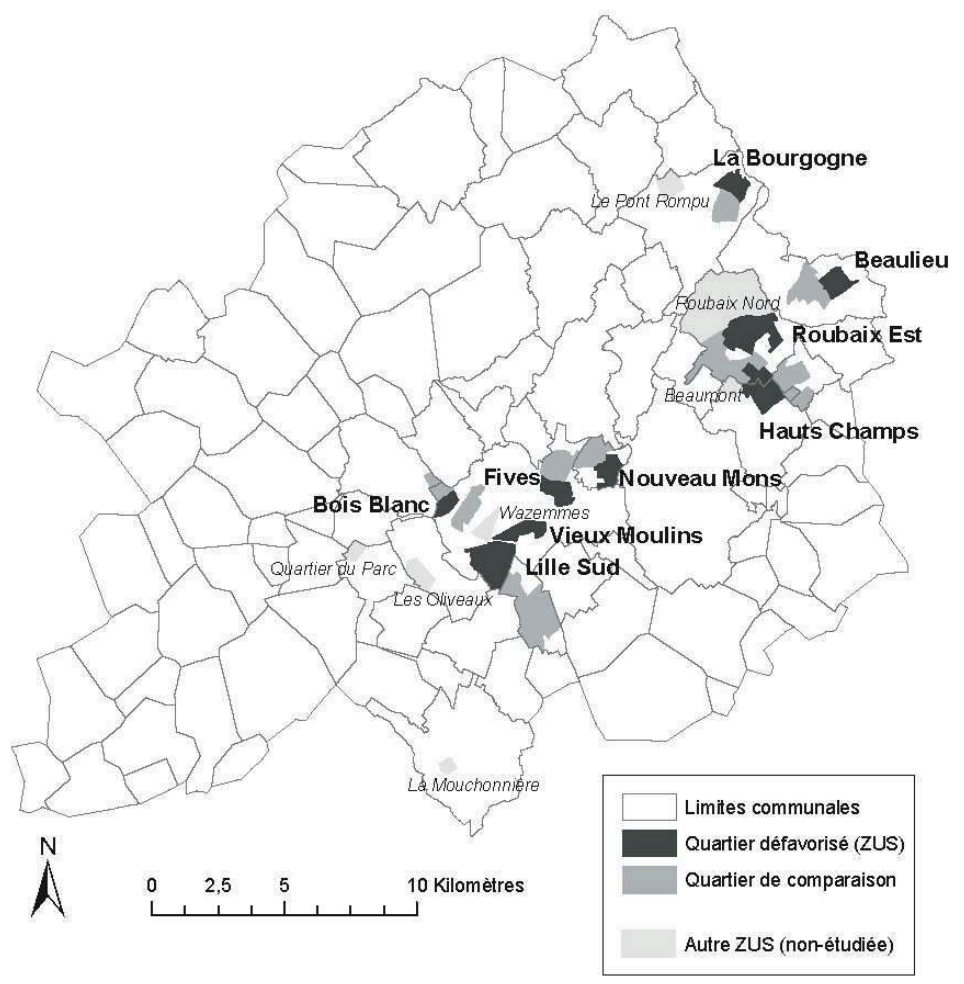

Réalisation : auteurs, 2015 est de $26 \%$ pour les quartiers de comparaison (d'après le Recensement Général de la Population, INSEE, 2007). Cet accès inégal à l'automobile se couple d'une plus faible mobilité : en moyenne, le nombre de déplacements quotidiens par habitant est de 3,1 pour les habitants des quartiers défavorisés contre 4,1 pour les habitants des quartiers de comparaison (d'après l'Enquête Ménages Déplacements, 2006). Les habitants des quartiers défavorisés se déplacent moins loin de leur domicile que les habitants des quartiers de comparaison (2,9 kilomètres contre 3,5 kilomètres en moyenne). Les habitants des quartiers défavorisés réalisent $45 \%$ de leurs déplacements à pied contre $34 \%$ des déplacements des habitants des quartiers de comparaison. En revanche, le nombre de déplacements à pied est similaire pour les deux types de quartiers avec un nombre moyen de 1,4 déplacement à pied par jour et par personne pour l'ensemble des quartiers étudiés.

\section{Un échantillon de 2185 habitants impliqués dans un accident}

Les données relatives aux accidents de la circulation sont confidentielles. Par l'intermédiaire du réseau TRANS-PV ${ }^{2}$, l'IFSTTAR $^{3}$ dispose cependant de l'ensemble des procès verbaux numérisés de 2000 à 2007. A partir des procès-verbaux d'accidents, on a pu différencier les individus impliqués dans un accident en tant que piéton et ceux impliqués dans un accident avec un autre mode de transport (conducteur ou passager de voiture particulière, cycliste, usager de transport en commun, etc.). Les procèsverbaux d'accidents permettent de connaitre l'adresse de résidence des personnes 
impliquées et de sélectionner ainsi les impliqués dans un accident qui habitent dans l'un des 18 quartiers d'étude.

Des informations sur certaines caractéristiques des personnes impliquées dans l'accident sont disponibles dans les procès-verbaux: en plus du sexe et de l'âge, l'activité professionnelle et la catégorie socioprofessionnelle sont renseignées. En l'absence d'autres données socio-économiques (comme le revenu ou le niveau d'éducation), nos analyses s'appuient donc sur (1) l'activité professionnelle qui distingue les actifs des chômeurs et personnes sans emploi ${ }^{4}$ et (2) la catégorie socioprofessionnelle qui différencie les cadres et professions intellectuelles supérieures des ouvriers et/ou employés.

17 Les habitants dont le sexe, l'âge, l'activité professionnelle, la catégorie socioprofessionnelle ou l'adresse de résidence ne sont pas renseignés sont exclus de l'analyse. Notre base de données regroupe ainsi 2185 individus ayant eu un accident entre 2000 et 2007 et habitant dans un des nos 18 quartiers d'étude. Parmi eux, 367 ont eu un accident en tant que piéton, soit $17 \%$ de l'effectif (Tableau 1).

18 Le contexte de résidence des personnes engagées dans les accidents est décrit à l'échelle des IRIS à partir de quatre variables différentes :

19 En lien avec l'environnement social : l'appartenance aux territoires institutionnels de la politique de la ville (appartenance à une Zone Urbaine Sensible). Cette variable propose une donnée synthétique de la pauvreté d'un quartier qui a été analysée en France en s'appuyant notamment sur des données relatives au chômage (Fleury et al., 2010; Haddak et al., 2012).

20 En lien avec l'habitat collectif : la part de logements en immeubles collectifs dans l'IRIS (d'après le Recensement Général de la Population de l'INSEE, 2007) analysée par la littérature anglo-saxonne (Mueller, et al., 1990).

21 En lien avec l'occupation des logements : le nombre moyen de personnes par pièce dans l'IRIS (d'après le Recensement Général de la Population de l'INSEE, 2007) également analysé par la littérature anglo-saxonne (Christoffel, et al., 1996; Anderson, et al., 1998).

Tableau 1 : Individus impliqués dans un accident de la circulation selon leur mode de transport et leur quartier de résidence

\begin{tabular}{l|rrr}
\hline & \multicolumn{3}{|c}{$\begin{array}{c}\text { Nombre d'impliqués (\%) dans } \\
\text { un accident de la circulation }\end{array}$} \\
& $\begin{array}{c}\text { Quartiers } \\
\text { défavorisés }\end{array}$ & $\begin{array}{c}\text { Quartiers de } \\
\text { comparaison }\end{array}$ & Total \\
\hline Piéton & $271(19 \%)$ & $96(12 \%)$ & $367(17 \%)$ \\
Cycliste & $39(3 \%)$ & $24(3 \%)$ & $63(3 \%)$ \\
Usager de deux-roues motorisés & $15(1 \%)$ & $7(1 \%)$ & $22(1 \%)$ \\
Passager de voiture particulière & $180(13 \%)$ & $123(16 \%)$ & $303(14 \%)$ \\
Conducteur de voiture particulière & $891(64 \%)$ & $539(68 \%)$ & $1430(65 \%)$ \\
\hline Total & $1396(100 \%)$ & $789(100 \%)$ & $2185(100 \%)$ \\
\hline
\end{tabular}

Réalisation : auteurs, 2015

22 En lien avec l'intensité du trafic routier : un indice de trafic défini comme la part de voies accueillant plus de 6000 véhicules par jour (hors autoroute) par rapport à l'ensemble 
des voies de circulation du quartier (données Lille Métropole Communauté Urbaine). La part des voies accueillant un fort trafic a été préférées au nombre de voies de plus de 6000 véhicules par jour dans la mesure où le maillage des quartiers et leur superficie sont très différents entre les IRIS. Cet indice permet d'obtenir une donnée homogène afin d'étudier les niveaux de trafic identifiés par la littérature (Macphersen et al., 1998; Christie et al., 2007).

\section{Utilisation de modèles de régression multiniveaux}

23 Des modèles de régression multiniveaux ont été utilisés afin d'étudier le rôle de variables au niveau de l'individu et de son contexte de résidence et de dissocier les contributions de ces différentes variables sur le risque d'être impliqué dans un accident en tant que piéton (Chaix \& Chauvin, 2002 ; Vallée, 2009). Une régression logistique est choisie dans la mesure où les données d'accidents sont des données discrètes (avoir un accident en tant que piéton/avoir un accident avec un autre mode de transport). Nous avons retenu le seuil de signification de $5 \%$. Pour chacun des modèles, les odds ratios et leur intervalle de confiance (IC) à $95 \%$ ont été calculés avec le logiciel Stata.

Différents modèles de régression sont réalisés. Ils intègrent - simultanément ou non les quatre variables individuelles (sexe, âge, activité professionnelle et catégorie socioprofessionnelle), et les trois variables contextuelles (statut ZUS / non-ZUS de l'IRIS de résidence, part des logements en immeuble collectif, nombre moyen de personnes par pièce et part des voies accueillant plus de 6000 véhicules par jour). Pour les variables relatives à l'habitat, à la sur-occupation et à l'intensité du trafic routier dans l'IRIS de résidence, les données ont été analysées selon le quartile, le premier quartile (le moins élevé) étant la catégorie de référence.

Ainsi, différents modèles ont été constitués: le modèle 1 intègre l'ensemble des variables individuelles, le modèle 2 le statut ZUS / non-ZUS de l'IRIS de résidence et le modèle 3 les variables individuelles et le statut de l'IRIS de résidence (Tableau 3). Pour étudier les associations avec les variables contextuelles, trois modèles ont été réalisés : le modèle 4 intègre les variables individuelles et la part des logements en immeubles collectifs, le modèle 5 les variables individuelles et le nombre moyen de personnes par pièce, et le modèle 6 les variables.

26 Pour chacun de ces modèles, la variance des effets aléatoires au niveau des IRIS est indiquée. Si cette variance est statistiquement supérieure à 0 , on peut considérer que le risque d'être impliqué dans un accident en tant que piéton varie de façon significative entre les IRIS de résidence.

\section{Facteurs individuels associés au risque d'accident piéton}

27 Les femmes impliquées dans un accident le sont davantage en tant que piéton que les hommes ( $23 \%$ des femmes impliquées dans un accident de la route sont des piétons contre $14 \%$ des hommes). Parmi l'ensemble des impliqués, la proportion d'individus ayant eu un accident en tant que piéton est forte pour les moins de 18 ans (52\%). Cette proportion diminue avec l'entrée dans l'âge de la conduite ( $7 \%$ pour les $18-24$ ans et $6 \%$ pour les $25-39$ ans) et augmente de nouveau avec l'âge ( $11 \%$ pour les $40-54$ ans, 
$14 \%$ pour les 55-64 ans) pour atteindre des valeurs importantes pour les personnes de 65 ans et plus ( $30 \%$ jusqu'à 79 ans et $52 \%$ pour les personnes de plus de 80 ans) (Tableau 2). Le risque accru d'accident piéton pour les habitants de 80 ans et plus s'explique par leur forte mobilité piétonne et par une moindre mobilité en voiture ou à vélo. Lorsqu'ils sont impliqués dans un accident de la route, les plus jeunes et les plus âgés le sont donc plus en tant que piéton que les autres classes d'âge de la population. Le Tableau 2 indique aussi que les taux d'impliqués en tant que piéton sont particulièrement élevés chez les scolaires et les retraités (avec des taux respectifs de $39 \%$ et de $30 \%)$. Ces résultats font écho aux résultats concernant l'âge. Enfin, notons que lorsqu'ils sont impliqués dans un accident, les chômeurs et les personnes sans emploi le sont plus en tant que piéton (11\%) que les actifs (6\%). Une hypothèse explicative est que les actifs sont moins souvent piétons que les personnes sans emploi. Les associations observées demeurent significatives après ajustement sur les autres variables individuelles (Modèle 1 du Tableau 4).

Ces résultats sont à mettre en regard avec les modes de transport utilisés. En effet, les femmes se déplacent davantage à pied que les hommes avec 1,4 déplacement par jour et par femme en 2006 dans l'agglomération lilloise contre 1,2 déplacement par jour et par homme (d'après l'Enquête Ménages-Déplacements Lille Métropole Communauté Urbaine, 2006). Les moins de 18 ans se déplacent également plus à pied que l'ensemble des autres classes d'âge avec 1,8 déplacement par jour et par personne contre 1,1 déplacement quotidien pour les plus de 18 ans. Les actifs marchent très peu avec 0,8 déplacements par jour et par personne contre 1,3 en moyenne. Ainsi, la forte implication des femmes et des moins de 18 ans dans les accidents en tant que piéton est à mettre en lien avec les modes de transport qu'ils utilisent quotidiennement.

Notons aussi que les lieux d'accidents des habitants des quartiers défavorisés sont plus proches de leur domicile que les lieux d'accidents des habitants des quartiers de comparaison (2,9 kilomètres contre 5,4 kilomètres) et que la part des accidents ayant eu lieu dans l'IRIS de résidence est plus forte (12,4\% contre $10,6 \%)$. Cette tendance s'explique par une mobilité plus proche du domicile pour les habitants des quartiers défavorisés.

\section{Facteurs contextuels associés au risque d'accident piéton}

\section{Un risque d'accident piéton plus élevé pour les habitants des ZUS}

30 Des différences sont observées selon le statut du quartier de résidence. Quand les habitants des quartiers défavorisés sont impliqués dans un accident, ils le sont plus en tant que piéton (19\%) que les habitants des quartiers de comparaison (12\%) (Tableau $\mathrm{n}$ o 4). Les habitants des IRIS appartenant à une Zone Urbaine Sensible ont 1,4 fois plus de risque d'avoir un accident en tant que piéton quand ils sont impliqués dans un accident (Modèle 2 du Tableau $n^{\circ} 3$ ).

31 Comme montré précédemment, la structure démographique de la population des quartiers varie entre les quartiers défavorisés et de comparaison : les proportions des jeunes et des personnes sans emploi y sont particulièrement élevées. Dans la mesure où ces catégories sont sur-impliquées dans les accidents piéton, l'écart de risque d'accident en tant que piéton entre les IRIS défavorisés et les IRIS non-défavorisés 
pourrait s'expliquer, du moins en partie, par la composition démographique des quartiers. Dans le modèle 3 où les variables sociodémographiques des individus sont prises en compte, on constate que le risque d'accident piéton demeure significativement plus élevé pour les habitants de quartiers défavorisés : les habitants des Zone Urbaine Sensible ont 1,5 fois plus de risque d'avoir un accident en tant que piéton quand ils sont impliqués dans un accident (modèle 3 du Tableau 3). Cet « effet ZUS » pourrait cependant être induit par un sous-ajustement du modèle au niveau individuel dans la mesure où certaines variables sociales inégalement répartis dans l'espace urbain, comme les revenus ou les niveaux d'éducation, n'ont pas pu être prises en compte.

Le modèle vide, autrement dit avant l'intégration des facteurs de risque, indique une variance significativement non nulle des résidus au niveau des IRIS pour le fait d'être impliqué en tant que piéton dans un accident. Le risque d'être impliqué dans un accident en tant que piéton varie donc de façon significative entre les IRIS de résidence. En observant l'évolution de la variance des résidus au niveau des IRIS, on constate que cette variance diminue dans les modèles qui intègrent le statut ZUS/non-ZUS des IRIS. Cette diminution est toutefois limitée car la variance des résidus au niveau des IRIS demeure non nulle. L'« effet ZUS» permet donc d'expliquer - mais seulement en partie - les inégalités spatiales de risque d'être impliqué dans un accident en tant que piéton.

Pour approfondir le rôle de cet "effet ZUS" sur le risque d'être impliqué dans un accident piéton d'une part et sur l'ampleur des disparités spatiales entre les IRIS de résidence, il est intéressant de prendre en considération d'autres variables relatives à l'habitat et au trafic dans le quartier de résidence. 
Tableau 2 : Risque d'être impliqué dans un accident en tant que piéton selon les variables individuelles

\begin{tabular}{|c|c|c|}
\hline & $\begin{array}{c}\text { Nombre } \\
\text { d'impliqués } \\
\text { dans un } \\
\text { accident }\end{array}$ & $\begin{array}{l}\text { Nombre d'impliqués } \\
(\%) \text { en tant que } \\
\text { piéton }\end{array}$ \\
\hline \multicolumn{3}{|l|}{ Sexe } \\
\hline Masculin & 1452 & $196(14 \%)$ \\
\hline Féminin & 733 & $171(23 \%)$ \\
\hline \multicolumn{3}{|l|}{ Age } \\
\hline Moins de 18 ans & 364 & $188(52 \%)$ \\
\hline de 18 à 24 ans & 511 & $37(7 \%)$ \\
\hline de 25 à 39 ans & 656 & $36(6 \%)$ \\
\hline de 40 à 54 ans & 395 & $43(11 \%)$ \\
\hline de 55 à 64 ans & 131 & $18(14 \%)$ \\
\hline de 65 à 79 ans & 97 & $29(30 \%)$ \\
\hline 80 ans et plus & 31 & $16(52 \%)$ \\
\hline \multicolumn{3}{|l|}{ Activité professionnelle } \\
\hline Ecolier, étudiants & 540 & $208(39 \%)$ \\
\hline Actif & 1100 & $68(6 \%)$ \\
\hline Chômeur /sans emploi & 374 & $40(11 \%)$ \\
\hline Retraité & 171 & $51(30 \%)$ \\
\hline \multicolumn{3}{|l|}{ Catégorie socioprofessionnelle } \\
\hline Artisans et chefs d'entreprise & 56 & $3(5 \%)$ \\
\hline Cadres et professions intellectuelles supérieures & 87 & $4(5 \%)$ \\
\hline Professions intermédiaires & 188 & $10(5 \%)$ \\
\hline Employés & 352 & $32(9 \%)$ \\
\hline $\begin{array}{l}\text { Ouvriers } \\
\text { Inactifs divers (chômeurs, retraités, personnes sans } \\
\text { emploi) }\end{array}$ & $\begin{array}{c}416 \\
1086\end{array}$ & $\begin{array}{c}19(5 \%) \\
299(28 \%)\end{array}$ \\
\hline \multicolumn{3}{|l|}{ Statut de l'IRIS de résidence } \\
\hline IRIS défavorisé (ZUS) & 1396 & $271(19 \%)$ \\
\hline IRIS de comparaison (non-ZUS) & 789 & $96(12 \%)$ \\
\hline
\end{tabular}

En gras : relation statistique significative au seuil de $5 \%$

Note de lecture : $14 \%$ des hommes impliqués dans un accident de la route sont des piétons.

Réalisation : auteurs, 2015 
Tableau 3 : Modélisation statistique du risque d'être impliqué dans un accident en tant que piéton selon les caractéristiques individuelles et le statut ZUS / non-ZUS de I'IRIS de résidence

\begin{tabular}{|c|c|c|c|c|}
\hline & Modèle vide & Modèle 1 & Modèle 2 & Modèle 3 \\
\hline & \multicolumn{4}{|c|}{ Odds Ratios (IC 95\%) } \\
\hline \multicolumn{5}{|l|}{ Sexe } \\
\hline Masculin & ' & Référence & ' & Référence \\
\hline Féminin & 1 & $1,9(1,5 ; 2,3)$ & 1 & $2,0(1,6 ; 2,5)$ \\
\hline \multicolumn{5}{|l|}{ Age } \\
\hline Moins de 18 ans & I & $17,2(11,8 ; 25,0)$ & ' & $19,2(12,8 ; 28,7)$ \\
\hline de 18 à 24 ans & I & $1,2(0,7 ; 1,8)$ & ' & $1,3(0,8 ; 2,1)$ \\
\hline de 25 à 39 ans & / & Référence & ' & Référence \\
\hline de 40 à 54 ans & 1 & $1,9(1,2 ; 3,0)$ & ' & $2,1(1,3 ; 3,4)$ \\
\hline de 55 à 64 ans & l & $3,4(2,0 ; 5,9)$ & l & $2,8(1,5 ; 5,2)$ \\
\hline de 65 à 79 ans & I & $7,9(4,6 ; 13,6)$ & ' & $7,9(4,5 ; 13,8)$ \\
\hline 80 ans et plus & 1 & $21,5(9,6 ; 48,1)$ & 1 & $21,5(9,5 ; 48,6)$ \\
\hline \multicolumn{5}{|l|}{ Activité professionnelle } \\
\hline Ecolier, étudiants & 1 & $9,4(7,0 ; 12,7)$ & l & $9,6(7,1 ; 13,1)$ \\
\hline Actif & l & Référence & l & Référence \\
\hline Chômeur /sans emploi & / & $1,7(1,1 ; 2,6)$ & ' & $1,8(1,2 ; 2,7)$ \\
\hline Retraité & 1 & $6,7(4,4 ; 10,1)$ & 1 & $6,7(4,4 ; 10,3)$ \\
\hline \multicolumn{5}{|l|}{ Catégorie socioprofessionnelle } \\
\hline Artisans et chefs d'entreprise & ' & $1,2(0,2 ; 5,4)$ & ' & $1,2(0,2 ; 5,4)$ \\
\hline $\begin{array}{l}\text { Cadres et professions intellectuelles } \\
\text { supérieures }\end{array}$ & l & Référence & I & Référence \\
\hline Professions intermédiaires & ' & $1,2(0,4 ; 3,9)$ & ' & $1,2(0,4 ; 3,9)$ \\
\hline Employés & / & $2,1(0,7 ; 6,0)$ & / & $2,1(0,7 ; 6,0)$ \\
\hline \multirow{2}{*}{$\begin{array}{l}\text { Ouvriers } \\
\text { Inactifs divers (chômeurs, retraités, } \\
\text { sans emploi) }\end{array}$} & l & $1(0,3 ; 2,9)$ & ' & $1(0,3 ; 2,9)$ \\
\hline & l & $7,9(2,9 ; 21,9)$ & / & $7,8(2,8 ; 21,6)$ \\
\hline \multicolumn{5}{|l|}{ Statut de l'IRIS de résidence } \\
\hline IRIS défavorisé (ZUS) & / & I & $1,4(1,1 ; 1,8)$ & $1,5(1,1 ; 2,1)$ \\
\hline IRIS de comparaison (non-ZUS) & 1 & 1 & Référence & Référence \\
\hline Variance inter-IRIS (IC 95\%) & $\begin{array}{c}0,12 \\
(0,04 ; 0,33)\end{array}$ & $\begin{array}{c}0,16 \\
(0,06 ; 0,45)\end{array}$ & $\begin{array}{c}0,07 \\
(0,02 ; 0,34)\end{array}$ & $\begin{array}{c}0,11 \\
(0,03 ; 0,43)\end{array}$ \\
\hline
\end{tabular}

En gras : relation statistique significative au seuil de $5 \%$

En gris : relation statistique non significative

Réalisation : auteurs, 2015

\section{Un risque d'accident piéton associé aux caractéristiques de l'habitat et du trafic dans le quartier de résidence ?}

\section{Un risque d'accident piéton plus fort pour les habitants des quartiers d'immeubles}

En moyenne, la part des logements en immeuble collectif est de $57 \%$ dans les quartiers défavorisés contre $42 \%$ dans les quartiers de comparaison. La pauvreté d'un IRIS est donc associée à une plus forte part de logements en immeubles collectifs.

La part de logements en immeuble collectif d'un IRIS n'est pas significativement corrélée (d'après le test de Student) à la part des habitants de cet IRIS impliqués en tant que piéton : en moyenne, la part d'immeubles des IRIS des habitants impliqués en tant que piéton est de $54 \%$ contre $52 \%$ pour les habitants impliqués avec un autre mode de transport. Une analyse par quartile ne montre pas d'association entre la part d'immeubles et les taux d'implication en tant que piéton (Tableau n ${ }^{\circ} 4$ ). En revanche, après ajustement sur les variables sociodémographiques des individus, on constate que les habitants des IRIS des deux derniers quartiles sont significativement plus impliqués en tant que piéton lorsqu'ils ont un accident (Modèle $4 a$ du Tableau ${ }^{\circ}{ }^{\circ}$ ).

Ce résultat est à mettre en relation avec l'étude de Mueller et al. En 1990 qui montrait que les enfants habitant dans un immeuble collectif avaient plus de risque d'avoir un accident (Mueller, et al., 1990). Notre analyse permet d'étendre ce résultat à l'ensemble de la population, et non uniquement les enfants, et de contrôler les variables individuelles et contextuelles. Une hypothèse explicative serait que les habitants de 
logements en immeuble collectif de quartiers défavorisés ne disposent pas d'espace extérieur en dehors de la rue et sont ainsi plus exposés au risque d'accident piéton.

Notons toutefois que la part de logements en immeuble collectif n'est plus significativement associé au risque d'être impliqué dans un accident en tant que piéton une fois que le statut ZUS / non-ZUS de l'IRIS de résidence est considéré (Modèle $4 \mathrm{~b}$ du Tableau n ${ }^{\circ}$ 5). On constate aussi que l'« effet ZUS » disparait avec l'intégration de la part de logements en immeubles collectifs dans le modèle (Modèle $4 \mathrm{~b}$ du Tableau $n{ }^{\circ} 5$ ). Ces deux variables contextuelles sont donc étroitement liées entre elles et leur association respective avec le risque d'accident en tant que piéton est difficile à démêler.

Tableau 4 : Risque d'être impliqué dans un accident en tant que piéton selon les variables contextuelles au niveau de l'IRIS de résidence

\begin{tabular}{|c|c|c|}
\hline & $\begin{array}{c}\text { Nombre } \\
\text { d'impliqués } \\
\text { dans un } \\
\text { accident }\end{array}$ & $\begin{array}{c}\text { Nombre } \\
\text { d'impliqués (\%) } \\
\text { en tant que } \\
\text { piétons }\end{array}$ \\
\hline \multicolumn{3}{|l|}{ Statut de I'IRIS } \\
\hline IRIS défavorisé (ZUS) & 1396 & $271(19 \%)$ \\
\hline IRIS de comparaison (non-ZUS) & 789 & $96(12 \%)$ \\
\hline \multicolumn{3}{|c|}{$\begin{array}{l}\text { Part des logements en immeubles } \\
\text { collectifs [quartiles] }\end{array}$} \\
\hline De 0 à $18,5 \%$ & 551 & $85(15 \%)$ \\
\hline De 18,6 à $59,1 \%$ & 547 & $73(13 \%)$ \\
\hline De 59,2 à $74,5 \%$ & 564 & $104(18 \%)$ \\
\hline De 74,6 à $99,6 \%$ & 523 & $91(17 \%)$ \\
\hline \multicolumn{3}{|c|}{$\begin{array}{l}\text { Nombre moyen de personnes par pièce } \\
\text { [quartiles] }\end{array}$} \\
\hline De 0,49 à 0,57 & 586 & $74(13 \%)$ \\
\hline De 0,58 à 0,64 & 580 & $95(16 \%)$ \\
\hline De 0,65 à 0,68 & 475 & $107(23 \%)$ \\
\hline De 0,69 à 1,14 & 544 & $91(17 \%)$ \\
\hline \multicolumn{3}{|l|}{ Indice de trafic [quartiles] } \\
\hline De 0 à $9,1 \%$ & 576 & $95(16 \%)$ \\
\hline De 9,2 à $18,6 \%$ & 523 & $86(16 \%)$ \\
\hline De $18,7 \%$ à $25,0 \%$ & 548 & $112(20 \%)$ \\
\hline De 25,1 à $51,5 \%$ & 538 & $61(11 \%)$ \\
\hline
\end{tabular}

En gras : relation statistique significative au seuil de $5 \%$

Note de lecture : $19 \%$ des habitants des ZUS impliqués dans un accident de la route sont des piétons. 
Tableau 5 : Modélisation statistique du risque d'être impliqué dans un accident en tant que piéton selon les caractéristiques de l'IRIS de résidence

\begin{tabular}{|c|c|c|c|c|c|c|}
\hline & Modèle $4 a^{*}$ & Modèle $4 b^{*}$ & Modèle $5 a^{*}$ & Modèle $5 b^{*}$ & Modèle $6 a^{*}$ & Modèle $6 b^{*}$ \\
\hline & \multicolumn{6}{|c|}{ Odds Ratios (IC 95\%) } \\
\hline \multicolumn{7}{|c|}{ Statut de I'IRIS de résidence } \\
\hline $\begin{array}{l}\text { IRIS défavorisé } \\
\text { (ZUS) }\end{array}$ & I & $1,2(0,9 ; 1,6)$ & l & $1,1(0,8 ; 1,6)$ & l & $1,3(1,0 ; 1,7)$ \\
\hline $\begin{array}{l}\text { IRIS de comparaison } \\
\text { (non-ZUS) }\end{array}$ & 1 & Référence & l & Référence & l & Référence \\
\hline \multicolumn{7}{|c|}{ Part des logements en immeubles collectif dans I'IRIS de résidence [quartiles] } \\
\hline De 0 à $18,5 \%$ & Référence & Référence & & & & \\
\hline De 18,6 à $59,1 \%$ & $1,1(0,7 ; 1,8)$ & $1,0(0,7 ; 1,5)$ & & & & \\
\hline De 59,2 à $74,5 \%$ & $2,0(1,3 ; 3,1)$ & $1,4(0,9 ; 2,0)$ & & & & \\
\hline De 74,6 à $99,6 \%$ & $1,7(1,1 ; 2,6)$ & $1,1(0,8 ; 1,6)$ & & & & \\
\hline \multicolumn{7}{|c|}{ Nombre moyen de personnes par pièce dans l'IRIS de résidence [quartiles] } \\
\hline De 0,49 à 0,57 & & & Référence & Référence & & \\
\hline De 0,58 à 0,64 & & & $1,3(0,9 ; 2,0)$ & $1,2(0,8 ; 1,7)$ & & \\
\hline De 0,65 à 0,68 & & & $1,5(1,0 ; 2,3)$ & $1,2(0,8 ; 1,8)$ & & \\
\hline De 0,69 à 1,14 & & & $2,2(1,5 ; 3,4)$ & $1,6(1,1 ; 2,4)$ & & \\
\hline \multicolumn{7}{|c|}{ Indice de trafic dans I'IRIS de résidence [quartiles] } \\
\hline De 0 à $9,1 \%$ & & & & & Référence & Référence \\
\hline De 9,2 à $18,6 \%$ & & & & & $0,9(0,6 ; 1,3)$ & $0,9(0,6 ; 1,4)$ \\
\hline De $18,7 \%$ à $25,0 \%$ & & & & & $1,1(0,7 ; 1,5)$ & $1,2(0,8 ; 1,7)$ \\
\hline De 25,1 à $51,5 \%$ & & & & & $1,0(0,7 ; 1,5)$ & $1,0(0,8 ; 1,5)$ \\
\hline $\begin{array}{l}\text { Variance inter-IRIS } \\
\text { (IC 95\%) }\end{array}$ & $\begin{array}{c}0,07 \\
(0,01 ; 0,37) \\
\end{array}$ & $\begin{array}{c}0,05 \\
(0,01 ; 0,44) \\
\end{array}$ & $\begin{array}{c}0,02 \\
(0,01 ; 1,62) \\
\end{array}$ & $\begin{array}{c}0,02 \\
(0,01 ; 2,77) \\
\end{array}$ & $\begin{array}{c}0,06 \\
(0,01 ; 0,42) \\
\end{array}$ & $\begin{array}{c}0,04 \\
(0,01 ; 0,68) \\
\end{array}$ \\
\hline
\end{tabular}

En gras : relation statistique significative au seuil de $5 \%$

En gris : relation statistique non significative

* Tous ces modèles intègrent le sexe, l'âge, l'activité professionnelle et la catégorie socioprofessionnelle des individus, de la variable contextuelle analysée (Modèle *a), ainsi que cette variable et le statut de I'IRIS de résidence (ZUS/non-ZUS) (Modèle *b).

Réalisation : auteurs, 2015

\section{Un risque d'accident piéton plus fort pour les habitants des quartiers aux logements sur-occupés}

En moyenne, la part des logements sur-occupés est de $68 \%$ dans les quartiers défavorisés contre $59 \%$ dans les quartiers de comparaison. La pauvreté d'un IRIS est donc associée à une plus forte part de logements sur-occupés.

Plus le nombre moyen de personnes par pièce est élevé dans un IRIS, plus ses habitants sont impliqués en tant que piéton. En moyenne, le nombre de personnes par pièce est de 0,7 pour les habitants impliqués en tant que piéton, contre 0,6 pour les habitants impliqués avec un autre mode de transport avec une différence significative (d'après le test de Student). Une analyse par quartile montre que les IRIS dont le nombre moyen de personnes par pièce est élevé comptent davantage d'habitants impliqués en tant que piéton, avec une baisse pour le dernier quartile : $13 \%$ pour le 1er quartile, $16 \%$ pour le 2nd quartile et $23 \%$ pour le 3ème quartile et $17 \%$ pour le dernier quartile (Tableau 4 ). Après ajustement sur les variables individuelles, les habitants des IRIS du quartile supérieur sont significativement plus impliqués en tant que piéton lorsqu'ils ont un accident (Modèle $5 \mathrm{a}$ du Tableau 5). Une très forte densité de personnes par logement dans l'IRIS de résidence est donc associée à un plus fort risque d'accident en tant que piéton. Ce résultat était connu pour les enfants (Christoffel et al., 1996), l'analyse permet de confirmer cette tendance pour l'ensemble de la population après contrôle des variables individuelles. Après ajustement sur les variables individuelles des 
individus et le statut ZUS / non-ZUS de l'IRIS de résidence, l'association entre les habitants impliqués en tant que piéton et la forte sur-occupation est significative, mais l'« effet ZUS » n'est plus statistiquement significatif (Modèle $5 b$ du Tableau 5).

L'analyse de la variance des résidus au niveau des IRIS indique aussi que la suroccupation des logements dans le quartier permet d'expliquer de façon optimale les différentiations spatiales observées entre les IRIS dans le risque d'être impliqué dans un accident en tant que piéton. La variance inter-IRIS mesurée dans les modèles intégrant la sur-occupation des logements dans le quartier est en effet plus faible que celle des modèles intégrant la part de logements en immeuble collectif et l'intensité du trafic routier

\section{Une absence d'association avec l'intensité du trafic routier dans le quartier de résidence}

41 En moyenne, la part des voies accueillant un trafic supérieur à 6000 véhicules par jour est de $17 \%$ dans les quartiers défavorisés contre $20 \%$ dans les quartiers de comparaison. Dans notre échantillon, les IRIS défavorisés sont donc moins traversés par des voies à forts trafics que les IRIS de comparaison.

42 L'intensité du trafic routier dans l'IRIS de résidence et le fait d'être impliqué en tant que piéton dans un accident ne sont pas corrélés (d'après le test de Student). Une analyse par quartile montre que la part des impliqués en tant que piéton parmi l'ensemble des impliqués augmente quand les niveaux de trafic augmentent ( $16 \%$ pour les deux premiers quartiles puis $20 \%$ pour le 3ème quartile), mais diminue fortement pour le dernier quartile ( $11 \%$ ) (Tableau 4). Après contrôle des variables individuelles, aucune association statistiquement significative n'apparait entre l'intensité du trafic routier du quartier de résidence et le risque d'accident en tant que piéton (Modèle 6a du Tableau 5). De même, après ajustement sur les variables individuelles des individus et le statut ZUS / non-ZUS de l'IRIS de résidence, aucune association statistiquement significative n'apparait entre les deux variables (Modèle $6 \mathrm{~b}$ du Tableau 5). La présence d'un fort trafic dans l'IRIS de résidence ne permet donc pas d'expliquer la différence de risque.

Ce résultat permet de nuancer l'influence du trafic routier dans le quartier de résidence comme facteur de risque d'accident piéton. Si les parents d'enfants qui habitent des quartiers défavorisés en Angleterre perçoivent le trafic comme un facteur de risque important (Christie et al., 2007), nos résultats ne montrent aucune association significative entre l'intensité du trafic dans le quartier de résidence et le risque d'accident piéton. Ainsi, le volume de circulation automobile, la hiérarchie routière et le nombre de branches aux intersections ont une incidence sur le nombre d'enfants blessés (Morency et al., 2011) lorsqu'on cible les lieux d'accidents, mais pas lorsqu'on étudie les lieux de résidence. Les niveaux de trafic dans le quartier de résidence ne permettent pas d'expliquer le risque supplémentaire pour les habitants des quartiers défavorisés d'être impliqué en tant que piéton. Ces résultats peuvent s'expliquer par la localisation des accidents à l'extérieur de l'IRIS de résidence. En effet, $78 \%$ des accidents impliquant un habitant d'un quartier défavorisé ont lieu à l'extérieur de leur IRIS de résidence. 


\section{Conclusion}

Cette étude a mis en évidence un cumul de facteurs individuels et contextuels pour comprendre le risque d'accident piéton. En effet, au niveau individuel, le risque d'accident piéton est accru pour les femmes, les moins de 18 ans et les plus de 80 ans, les chômeurs et les personnes sans emploi. Au niveau contextuel, quand ils sont impliqués dans un accident, les habitants de quartiers défavorisés le sont davantage en tant que piéton que les habitants des quartiers favorisés. Cette différence entre quartiers n'est pas le simple reflet de la structure par âge ou par sexe de la population ni de sa composition sociale. En effet, après ajustement sur la structure démographique et sociale de la population, les habitants d'IRIS défavorisés ont un risque 1,5 fois plus élevé d'être impliqué en tant que piéton quand ils ont un accident. Des caractéristiques de l'environnement résidentiel sont également associées à un plus fort risque d'accident piéton : la forte présence de logements en immeubles collectifs et une suroccupation des logements dans l'IRIS de résidence sont associés à un fort risque d'accident piéton. En revanche, aucune association n'a été mise en évidence avec l'intensité du trafic routier dans le quartier de résidence.

La question des modes de transports utilisés par les habitants a été mobilisée comme élément explicatif des risques d'accidents. Mais, il aurait été intéressant d'intégrer l'usage de ces modes aux analyses afin de contrôler les associations observées entre le risque d'accident piéton et les caractéristiques de l'environnement de résidence avec les distances parcourues à pied par les personnes impliquées dans les accidents. Intégrer des données sur la mobilité et les distances parcourues par mode de transport aurait permis d'analyser plus précisément l'exposition au risque d'accident piéton et les inégalités socio-spatiales associées. Toutefois, l'échantillonnage de l'Enquête Ménages-Déplacements ne permettait pas de telles analyses. Une enquête ad hoc sur ce thème approfondirait les liens entre le risque d'accident (notamment piéton) et les comportements de mobilité des populations.

\section{BIBLIOGRAPHIE}

Abdalla I.M., Raeside R., Barker D., Mcguigan D.R.D., 1997, An investigation into the relationships between area social characteristics and road accident casualties. Accident Analysis and Prevention. Vol. 29, $\mathrm{n}^{\circ}$ 5, pp. 583-593.

Anderson C.L., Agran P.F., Winn D.G., Tran C., 1998, Demographic risk factors for injury among Hispanic and non-Hispanic white children : an ecologic analysis. Injury Prevention. Vol. 4, pp. 33-38.

Chaix B., Chauvin P., 2002, L'apport des méthodes d'analyse multiniveau dans l'analyse contextuelle en épidémiologie sociale : une revue de la littérature. Revue d'Epidemiologie et de Santé Publique. Vol. 50, pp. 489-499. 
Christie N., Ward H., Kimberlee R., Towner E., Sleney J., 2007, Understanding high traffic injury risks for children in low socioeconomic areas: a qualitative study of parents' views, Injury Prevention, $\mathrm{n}^{\circ}$ 13, pp. 394-397.

Christoffel K.K., Donovan M., Schofer J., 1996, Psychosocial factors in childhood pedestrian injury: a matched casecontrol study. Pediatrics. Vol. 97, pp. 33-42.

Cloutier M.-S., Tremblay M., Morency P., Apparicio P., 2014, Carrefours en milieu urbain : quels risques pour les piétons ? Exemple empirique des quartiers centraux de Montréal, Canada. Recherche Transports Sécurité, Vol. 30, pp. 3-20.

Dougherty G., Pless B., Wilkins R, 1990, Social Class and the Occurrence of Traffic Injuries and Deaths in Urban Children. Canadian Journal of Public Health, Vol. 81, pp. 204-209.

Edwards P., Green J., Roberts I., Grundy C., 2006, Deprivation and road safety in London : A report to the London Road Safety Unit. Londres : London School of Hygiene \& Tropical Medicine.

Fleury D., Peytavin J.-F., Alam T., Godillon S., Saint Gérand Th., Medjkane M., Millot M., 2010, Inégalité sociale et Risque Routier. L'apport d'une approche territorialisée. Cahiers Scientifique du Transport, Vol. 57, pp. 45-62.

Fol S., 2009, La mobilité des pauvres, Paris : Belin.

Haddak M., Pochet P., Licaj I., Randriantovomanana E., Vari J., Mignot D., 2012, Inégalités sociales de risque routier et mobilité à l'adolescence, In Carnis L., Mignot D. (Dir), Pour une économie de la sécurité routière, Emergence d'une approche pour l'élaboration de politiques publiques, Paris : Economica coll « Méthodes et approches », pp. 99-120.

Hasselberg M., Laflamme L., Ringback Weitofbt G., 2001, Socioeconomic differences in road traffic injuries during childhood and youth: a closer look at different kinds of road user. Epidemiology \& Community Health. Vol. 55, pp. 858-862.

Macpherson A., Roberts I., Pless I.B., 1998, Children's exposure to traffic and pedestrian injuries. American Journal of Public Health. Vol. 88, $n^{\circ}$ 12, pp. 1840-1843.

Morency P., Gauvin L., Tessier F., Miranda-Moreno L., Cloutier, M.-S., Morency, C., 2011, Analyse désagrégée des facteurs environnementaux associés au nombre d'enfants blessés par un véhicule à moteur en milieu urbain. Cahiers de géographie du Québec, vol. 55, $\mathrm{n}^{\circ}$ 156, pp. 449-468.

Mueller B.A., Rivara F.P., Lii S.M., 1990, Environmental factors and the risk for childhood pedestrian-motor vehicle collision occurrence. American Journal of Epidemiology. Vol. 132, pp. 550-560.

ONISR - Observatoire Nationale de la Sécurité Routière, 2014, Bilan de la sécurité routière - pré édition 2013, consulté en janvier 2015, http://www.securite-routiere.gouv.fr/la-securiteroutiere/l-observatoire-national-interministeriel-de-la-securite-routiere/bilans-annuels/lesbilans-annuels-de-la-securite-routiere-en-france

Reimers A., Ponce de Leon A., Laflamme L., 2008, The area-based social patterning of injuries among 10 to 19 year olds Changes over time in the Stockholm County. BMC Public Health. Vol. 8, pp. 131-142.

Roberts I., Power C., 1996, Does the decline in child injury mortality vary by social class? A comparison of class specific mortality in 1981 and 1991. British Medical Journal, Vol. 313, pp. 784-786.

Sonkin B., Edwards P., Roberts I., Green J., 2006, Walking, cycling and transport safety: an analysis of child road deaths. Journal of the Royal Society of Medecine. Vol. 99, pp. 402-405. 
Vallée J, 2009. Les disparités spatiales de santé en ville. L'exemple de Vientiane (Laos). Cybergeo Revue Européenne de géographie. Article 477. http://www.cybergeo.eu/index22775.html

Van Beeck E., Mackenbach J.P., Looman C.W.N., 1991, Determinants of traffic accident mortality in the Netherlands: a geographical analysis. International Journal of Epidemiology. Vol. 20, pp. 698-706.

Quetelard B., 1998, La mobilité dans les quartiers de la politique de la ville : enseignements des enquêtes ménages déplacements de Lille (1987) et Lyon (1995), In Ministère de l'Equitement, du Transport et du logement, Les transports et la ville, analyses et diagnostics, Paris : Presse de l'Ecole Nationale des Ponts et Chaussées, pp. 34-38.

\section{NOTES}

1. Un IRIS est un découpage de l'INSEE. Ces «Ilots Regroupés pour l'Information Statistique » font référence à la taille visée de 2000 habitants.

2. Trans-PV est un organisme dépendant des assureurs, chargé d'envoyer aux compagnies d'assurance concernées un exemplaire du PV pour l'indemnisation des victimes.

3. Les résultats de cet article sont extraits d'une thèse réalisée au département Mécanismes d'Accidents de l'IFSTTAR (Institut français des sciences et technologies des transports, de l'aménagement et des réseaux). Les auteurs remercient ce département pour l'accès aux données.

4. Cette catégorie regroupe une grande hétérogénéité de situation entre des personnes au chômage entre deux emplois, des personnes au chômage longue durée, des personnes au foyer, etc. Le codage des PV ne permet pas de distinguer des souscatégories.

\section{RÉSUMÉS}

Pour approfondir l'analyse des inégalités socio-spatiales devant le risque d'accident de la route, cet article s'intéresse aux caractéristiques individuelles et contextuelles des personnes impliquées dans un accident en tant que piéton. En analysant les données relatives aux accidents dans l'agglomération lilloise à l'aide de modèles de régression statistique multiniveaux, il s'agit de comparer le profil des individus impliqués dans un accident en tant que piéton avec celui des individus impliqués dans un accident avec un autre mode de transport. Les résultats montrent que, quand ils sont impliqués dans un accident de la route, les habitants des Zones Urbaines Sensibles (ZUS) le sont davantage en tant que piéton que les habitants d'autres quartiers. Cette différence n'est pas le simple reflet de la structure démographique et sociale des ZUS puisqu'elle demeure significative après ajustement sur le sexe, l'âge, l'activité professionnelle et la catégorie socioprofessionnelle des individus. D'autres caractéristiques du quartier de résidence - comme une forte présence de logements en immeubles collectifs et une sur-occupation des logements - 
se révèlent associées à un risque accru d'accident piéton. En revanche, aucune association n'a été mise en évidence avec l'intensité du trafic routier dans le quartier de résidence.

According to the literature, social and socio-spatial inequalities exist in traffic accidents. Research indicates that road accident rates are particularly high for low-income classes (Van Beeck et al., 1991; Hasselberg, et al., 2001). Other studies have focused on the characteristics of the residential areas in which victims of accidents live, and these also show that accident rates are higher for residents in poorer areas (Dougherty et al., 1990; Abdalla et al., 1997; Fleury et al., 2010; Haddak et al., 2012). Certain types of road users, like pedestrians, are especially vulnerable. France's National Observatory of Road Safety (Observatoire National de la Sécurité Routière) has found that the risk of being seriously injured is 1.6 times higher for pedestrians than people travelling by car (ONISR 2014). Given the trend to increased walking as a mode of transport in cities, it is particularly important to understand better the risks pedestrians face when trying to improve road safety.

This article investigates the profile of individuals as pedestrians who are involved in accidents by comparing them with persons who experienced accidents arising in other forms of transport (drivers or passengers of personal vehicles, cyclists, people using public transport, etc.). The aim is to analyse the socio-spatial inequalities of risks faced by pedestrians: are persons living in poorer areas likelier to be involved in road accidents than people living in richer areas? Once the individual characteristics are taken into account, what are the variables of the residential environment that are associated with an increased risk of having a pedestrian accident? To what extent are the characteristics of an individual's residential area (in terms of social housing or traffic environment) linked to the risk of being involved in a traffic accident as a pedestrian?

This research focuses on road accidents involving residents of nine disadvantaged neighbourhoods in the Lille area, in northern France. According to the inventory of accidents recorded by the police, 2,185 residents in these areas were involved in a road traffic accident between 2000 and 2007 . Around $17 \%$ of these persons were pedestrians. Other databases provide information about the residential neighbourhood of the victims, including a population census, a mobility survey and traffic data. The methodology here uses a multi-level regression model. To build this model, demographic and social characteristics of the inhabitants are used (including sex, age, activity and socio-professional category). Information relating to residential areas is also used, such as the share of housing units in apartment flats, the average number of persons per room and road traffic volume.

The results show that several individual and contextual factors come into play in understanding the risks of pedestrian accidents. At the individual level, risks are higher for women, persons under 18 , and persons over 80 , as well as for the unemployed and people without work. From a contextual point of view, accident victims in poorer neighbourhoods are likelier to be pedestrians than are the residents of richer areas. This difference between areas is not merely a reflection of the age or sex structure of the population, nor its social composition. Once adjustments have been made to take into account the demographic and social structure of a population, the inhabitants of poorer areas have a risk 1.5 times greater of being involved in accident as pedestrians (see Table). The characteristics of the residential environment are also associated with a higher risk of a pedestrian accident. These include a strong proportion of housing units in apartment flats, and high housing occupation rates. No evidence was found with respect with the traffic intensity in the residential area.

\section{INDEX}

Mots-clés : risques, accident, piéton, inégalités sociales et territoriales 
AUTEURS

SYLVANIE GODILLON

Géographe, docteur associée - UMR Géographie-cités

JULIE VALLÉE

Géographe, chargée de recherche au CNRS -UMR Géographie-cités 